\title{
Impacts of the Staphylococcal Enterotoxin H on the Apoptosis and IncRNAs in PC3 and ACHN
}

\author{
M. Safarpour-Dehkordi ${ }^{a}$, A. Doosti ${ }^{a, *}$, and M. S. Jami ${ }^{b, c}$ \\ ${ }^{a}$ Department of Biology, Faculty of Basic Sciences, Shahrekord Branch, Islamic Azad University, Shahrekord, Iran \\ ${ }^{b}$ Department of Neurology, David Geffen School of Medicine, University of California Los Angeles (UCLA), \\ CA 90095, United States \\ ${ }^{c}$ Cellular and Molecular Research Center, Basic Health Sciences Institute, \\ Shahrekord University of Medical Sciences, Shahrekord, Iran \\ *e-mail: Abbasdoosti@yahoo.com \\ Received December 20, 2019; revised January 18, 2020; accepted January 31, 2020
}

\begin{abstract}
Cancer is considered as the most lethal disease for human beings, and up to now many attempts were failed for prevention and treatment of this tremendous health problem. Consequently, this study purpose was to investigate novel therapeutic methods for cancer. The bacterial toxin can result in cell death throughout the induction of apoptosis in cancer cell lines. We evaluated apoptosis and the expression levels of long non-coding RNAs (lncRNAs) in PC3, ACHN and HDF cell lines that were transfected with pCDNA3.1(+)-seh and empty plasmid. pCDNA3.1(+)-seh treatment showed overexpression of $G A S 5(p=0.0033$ and $p=0.0033)$ in PC3 and ACHN cells, down regulation of PCA3 and NEAT1 $(p=0.0092$ and $p=0.0097)$ in the PC3 cells, and down regulation of PVT1 and MALAT1 $(p=0.0239$ and $p=0.0133)$ in the ACHN cells in comparison with the empty plasmid, but there was no significant effect on HDF normal cells. Additionally, this study data demonstrated that the cell adhesion was down regulated. The flow cytometry data showed transfection by pCDNA3.1 (+)-seh could elevate PC3 and ACHN cell apoptosis levels in comparison with empty plasmid. This study findings propose that SEH toxin of $S$. aureus could be a useful candidate for therapeutic researches in cancer vaccine development.
\end{abstract}

Keywords: apoptosis, lncRNA, prostate cancer, kidney cancer, SEH toxin

DOI: $10.3103 /$ S0891416820030076

\section{INTRODUCTION}

Cancer is a generic term for a large group of diseases that represent the second leading cause of death worldwide. Prostate cancer $(\mathrm{PCa})$ is one of the major forms of cancer among males, and many studies have shown that the incidence of $\mathrm{PCa}$ in recent years has grown substantially [1]. The global burden of PCa is considerable, ranking among the top five cancers for both incidence and mortality. In addition, $\mathrm{PCa}$ is the second leading cause of cancer death among men after lung cancer [2]. The combined treatment, applying both local and systemic therapies, can be beneficial in the management of prostate cancer. More specifically, treatment approaches like surgery, radiation therapy, hormonal therapy, and chemotherapy are used for prostate cancer. Immunotherapy has also offered new possibilities to treat prostate cancer with metastases that does not respond to other forms of treatment [3]. The next most common cancers is kidney cancer. The most common form of kidney cancer originates in the renal epithelium, called renal cell carcinoma (RCC). The classification system of kidney tumors has recently been updated, with clear cell, papillary (types
I and II), and chromophobe tumors being the most frequent RCC subtypes encompassing $85 \%$ of all primary renal neoplasms. About $25-30 \%$ of RCC patients are diagnosed when they are already at advanced stages of this cancer [4]. RCC is the 9th and 14th most common cancer in men and women, respectively, and it is the 16th most common cause of cancer death from cancer worldwide. In light of improved screening programs of patients with RCC, we have learned that the prevalence of this cancer is increasing worldwide. The highest incidence rates can be found in developed countries, mostly Northern and Eastern Europe, such as the Czech Republic, as well as North America [5, 6]. In the US, there are 64000 new cases of RCC and 14000 RCC-related deaths every year. The important factors that play a role in this disease are race, age, and gender. In men above the age of 60 , $\mathrm{RCC}$ is more common, and the highest incidence is in the 60-80 decades of life [4]. The genetic and acquired risk factors comprise multiple agents causing RCC. Smoking, hypertension, obesity, diabetes, and chronic analgesic use are the most common acquired risk factors for this cancer [7]. Cell lines are still a use- 
ful tool for many biological and biomedical studies, including cellular biology and oncology, and the proper use of in vitro models plays a central role in enhancing our knowledge of cancer. The ACHN cell line indicates an uncertain RCC histotype. It was established from the malignant pleural effusion of a 22-year-old Caucasian male with widely metastatic renal adenocarcinoma [8].

Bacterial toxins have a great therapeutic potential for cancer therapy. In several studies (in vitro and in vivo), these toxins showed efficient cell-killing capacity for cancer cells. A number of bacterial toxins such as Staphylococcus aureus alpha-toxin ( $\alpha$-toxin), Clostridium perfringes enterotoxin, and streptolysin O of Streptococcus pyrogenes are the most attractive for cancer therapy. Bacterial toxins are the most obvious cytotoxic agents because these genes are native to bacterial physiology. Apoptosis is induced by bacterial toxins during infection and is now considered important in disease processes $[9,10]$. As a major human pathogen, $S$. aureus also causes apoptosis during infection. In some diseases like atopic dermatitis and sepsis, the $S$. aureus influences the severity and result of a disease by inducing apoptosis. Some studies reported that most of the $S$. aureus toxins under examination triggered programmed cell death processes. For example, staphylococcal enterotoxin $\mathrm{H}$ (SEH) triggered apoptosis in bovine mammary epithelial cells. Several $S$. aureus toxins, such as staphylococcal enterotoxins (SEs) and a-toxin, have proapoptotic activities. The SEs include at least 10 members: SEA, SEB, SEC, SED, SEE, SEG, SEH, SEI, SER, and SET [11].

Almost thirty years ago, the first long non-coding RNAs (lncRNAs) were discovered and characterized for their involvement in genomic imprinting and chromosome dosage-compensation. Now, about 30000 lncRNAs have been identified in the human genome. lncRNAs have the major transcriptional output of the genome; they are not responsible for encoding any proteins and are defined as RNA molecules with more than 200 nucleotides. studies have found that these transcripts played a central role in several physiological and pathological processes. IncRNAs also play important roles in cancer, they are often differentially expressed and widely associated with numerous types of cancer. The aberrant expression of these transcripts has shed light on tumorigenesis, metastasis, cancer stage progression, and patient survival. Hence, lncRNas have been used for diagnostic and therapeutic approaches [12].

In this study for the first time, we inserted seh gene in the pcDNA3.1(+) eukaryotic expression plasmid to construct a recombinant carrier. We transfected ACHN and PC3 cell lines with the carrier and empty plasmid in vitro and evaluate the expression of desired lncRNAs in the cells and apoptosis.

\section{MATERIALS AND METHODOLOGY}

IncRNAs Signature Selection with the Highest Gene Expression Alteration Score amongst Those with Prostate and Kidney Cancers

In order to indicate the contribution of IncRNAs in prostate and kidney cancers, 4105 lncRNAs were downloaded from the HUGO Gene Nomenclature Committee (HGNC) database (http://www.genenames.org). All the genes were recognized using the cBioPortal after we individually input them (http://www.cbioportal.org). We selected the GAS5, PCA3 and NEAT1 for prostate cancer and GAS5, PVT1 and MALAT1 1ncRNAs for kidney cancer with RNA seq v2 data in this study, respectively, and also considered RNA expression Z score threshold as \pm 2 .

\section{Recombinant Plasmid Preparation and Confirmation}

The mammalian expression vector, pcDNA3.1(+) containing seh, encoding gene was purchased from GenRay Biotechnology (Dongjing town Songjiang, Shanghai China), and an empty pcDNA3.1(+) (Invitrogen) plasmid was utilized in this program. Both plasmids were transformed into two separate competent Escherichia coli strain TOP10F cells using heat shock method [13], and were grown under ampicillin $(100 \mu \mathrm{g} / \mathrm{mL})$ treatment. The recombinant and empty plasmids were extracted by the use of the Plasmid MiniPrep Purification Kit (Qiagen, Germany) with respect to the manufacturer's protocol. The recombinant vector (pcDNA3.1 (+)-seh) was analyzed using specific-seh PCR primers. In addition, this vector was digested using the restriction enzymes BamHI and EcoRV (both from New England BioLabs, USA) in order to confirm the seh gene presence in the recombinant plasmid. The PCR amplified and digested products were electrophoresed on $1 \%$ agarose gel.

\section{Cell Transfection}

ACHN (human renal cell adenocarcinoma), PC3 (human prostate adenocarcinoma cell line) and HDF (human dermal fibroblasts-normal) cell lines were purchased from the National Cell Bank of Iran (Pasteur Institute, Tehran, Iran). The cells were cultured and maintained using RPMI-1640 medium (SigmaAldrich, St. Louis, MO, USA) with $10 \%$ heat-inactivated fetal bovine serum (FBS) (Gibco, Carlsbad, CA, USA), $\quad 100 \mathrm{U} / \mathrm{mL}$ penicillin (Sigma-Aldrich, St. Louis, MO, USA), and $100 \mathrm{ug} / \mathrm{mL}$ streptomycin (Sigma-Aldrich, St. Louis, MO, USA) in a humidified atmosphere containing $5 \%$ carbon dioxide $\left(\mathrm{CO}_{2}\right)$ at $37^{\circ} \mathrm{C}$. The transfection of cells was performed in 6-well plate with respect to the instructions for the Lipofectamine $2000^{\mathrm{TM}}$ reagent (Invitrogen, USA). $2 \mu \mathrm{g}$ of pcDNA3.1 (+)-seh and $2 \mu \mathrm{g}$ of the pcDNA3.1 $(+)$ were separately transfected into each cell lines. The transfected ACHN, PC3 and HDF cells were 
selected with $600 \mu \mathrm{g} / \mathrm{mL}$ G418 (Invitrogen, USA) [14]. In addition, there exist one group from each cells, which were cultured in the same condition in 6-well plate without any transfection. These cells were treated with G418, similar to control groups in order to assess this aminoglycoside antibiotic accurate performance. HDF cells were applied as normal cells group during this experiment.

\section{Annexin V-FITC Assay}

Apoptotic cell death caused by recombinant (pcDNA3.1(+)-seh) and empty plasmid (pcDNA3.1(+)) was measured by the use of FITC Annexin V Apoptosis Detection Kit I (556547, BD Biosciences Pharmingen, USA) by flow cytometry. All experiments were accomplished in duplicate; briefly explain, $3 \times 10^{5}$ of each cell lines (ACHN, PC3 and HDF) were two times washed with ice-cold phosphate buffer solution. After that, the cells were re-suspended in $100 \mu \mathrm{L}$ of $1 \mathrm{X}$ binding buffer (provided in the kit), and also $100 \mu \mathrm{L}$ of cells suspension was transferred into flow cytometry micro-tube and was stained with $5 \mu \mathrm{L}$ of FITC-conjugated Annexin-V (10 mg/mL) and $10 \mu \mathrm{L}$ of PI $(50 \mathrm{mg} / \mathrm{mL})$. The cells were incubated in darkness for $15 \mathrm{~min}$ at room temperature $\left(25^{\circ} \mathrm{C}\right)$. Afterward, $400 \mu \mathrm{L}$ of binding buffer was added, and analyzed using a flow cytometry apparatus (FACSCalibur, Becton. Dickinson).

\section{$R N A$ Isolation and $c D N A$ Synthesis}

Cells were collected after being transfected by a recombinant plasmid and empty plasmid for duration of 10 days. Total RNA was extracted using RNX-Plus reagent (SinaClon, Iran). After that, RNA was dissolved in sterile RNase-free water. The RNA concentration and purity were measured using a spectrophotometer (Thermo Scientific ${ }^{\mathrm{TM}}$ NanoDrop 2000, Germany). The complementary DNA (cDNA) synthesis was accomplished using PrimeScript ${ }^{\mathrm{TM}}$ First-Strand cDNA Synthesis kit (cat no. 6610A; Takara Biomedical Technology, China).

Also, a PCR test was applied by the use of seh specific primers, in order to confirm the seh gene expression after lipoprotection. PCR was performed in a $20 \mu \mathrm{L}$ mixture containing $1 \mu \mathrm{L} \mathrm{cDNA}, \mathrm{MgCl}_{2} 2 \mathrm{mM}$, dNTPs $0.2 \mathrm{mM}$, Taq DNA polymerase 0.5 unit (SinaClon), and $0.5 \mu \mathrm{M}$ of each primer (seh-F/seh-R, Table 1). The reaction mixture was heated for $3 \mathrm{~min}$ at $95^{\circ} \mathrm{C}$. Amplification was performed in a Bio-rad T 100 Thermal Cycler (USA), at $94^{\circ} \mathrm{C}$ for $1 \mathrm{~min}, 55^{\circ} \mathrm{C}$ for $1 \mathrm{~min}, 72^{\circ} \mathrm{C}$ for $1 \mathrm{~min}$ for 30 cycles, respectively, followed by a final extension step for $5 \mathrm{~min}$ at $72^{\circ} \mathrm{C}$. The PCR products were analyzed on a $1 \%$ agarose gel using electrophoresis.

\section{qPCR Analysis}

Real time RT-PCR was performed using specific primers (Table 1) and SYBR ${ }^{\circledR}$ Premix Ex TaqTM II kit (TaKaRa, Japan), with respect to the manufacturer's instruction. The qPCR analysis was performed by specific primers of GAS5, PCA3 and NEAT1 lncRNAs on PC 3 cDNA. Correspondingly, the PVT1, MALAT1 and $G A S 5$ lnc RNAs expression levels were evaluated in ACHN cells, and also Bax and Bak genes were evaluated as pro-apoptotic genes on HDF cells, as normal group. All samples were amplified in triplicate at the same PCR condition and amplification was separately performed for each target gene. No template controls (NTC) that contains DNAse-free water instead of template cDNA, were also included in each run. Realtime RT-PCR was accomplished using a Rotor-Gene 6000 Real-Time PCR Machine (Qiagen, Germany). The thermal cycling conditions were as followings: $95^{\circ} \mathrm{C}$ for $2 \mathrm{~min}$, and $45 \mathrm{cycles}$ of $95^{\circ} \mathrm{C}$ for $20 \mathrm{~s}, 60^{\circ} \mathrm{C}$ for $15 \mathrm{~s}$ and $72^{\circ} \mathrm{C}$ for $20 \mathrm{~s}$, respectively. In addition, a melting curve analysis was applied in order to ensure about correct amplification of the expected amplicons, after the qPCR run. Standard curves for $G A P D H$ was generated using cDNA serial dilution. GAPDH was monitored as a reference gene and specific gene expression level was normalized in terms of $G A P D H$ transcript, and was calculated using $2^{-\Delta \mathrm{Ct}}$ method. Also, the PCR products were electrophoresed on $2 \%$ agarose gel in order to verify the product sizes.

\section{Cell Adhesion Assay}

In order to determine the cells adhesion to the flask bottom, a real time RT-PCR assay was performed for investigating the integrin gene expression using specific primers. In addition, real time RT-PCR for $\beta$-catenin gene was performed in order to determine the cell to cell sticking. At this stage, the qPCR was performed as it was explained earlier.

\section{Statistical Analysis}

All information were presented as mean \pm SEM. Paired Student T-test was conducted for statistical analysis. The differences with less than $0.05 p$-value were considered as the statistical significance level. GraphPad Prism (v8; GraphPad software) was applied for accomplishing the aforementioned statistical analysis.

\section{RESULTS \\ IncRNAs Were Found in Cancers}

At first, we downloaded all approved lncRNAs from HGNC to uncover the roles of IncRNAs in prostate and kidney cancer. With the 4105 lncRNAs that were approved, we investigated the cBioPortal database and found lncRNAs obvious alterations, including: mutation, copy number alteration and expression. 
Table 1. The primers used in real time RT-PCR analysis

\begin{tabular}{|c|c|c|c|c|}
\hline Genes & $\begin{array}{l}\text { Primers } \\
\text { name }\end{array}$ & Sequences & $\begin{array}{c}\text { Annealing } \\
\text { temperature, }{ }^{\circ} \mathrm{C}\end{array}$ & $\begin{array}{l}\text { Product } \\
\text { length, bp }\end{array}$ \\
\hline$\overline{\text { Seh }}$ & $\begin{array}{l}\text { Seh-F } \\
\text { Seh-R }\end{array}$ & $\begin{array}{l}\text { 5-AGTGCGAGAAGATCAGCGAGAAC-3 } \\
\text { 5-ATCTTCCGAATCTTAATGTCCAGC-3 }\end{array}$ & 65 & 193 \\
\hline GAS5 & $\begin{array}{l}\text { GAS5-F } \\
\text { GAS5-R }\end{array}$ & $\begin{array}{l}\text { GAS5-F: 5'-ATTGGCACACAGGCATTAGACAG-3' } \\
\text { GAS5-R: 5'-CTTCTTTAAAACTTGCTCCACACAG-3' }\end{array}$ & 64 & 206 \\
\hline MALAT1 & $\begin{array}{l}\text { MALAT1-F } \\
\text { MALAT1-R }\end{array}$ & $\begin{array}{l}\text { MALAT1-F: 5'-TTCGGAGACAAAGCCATTCGC-3' } \\
\text { MALAT1-R: 5'-TTTCTACCGTTTTTCAGCTTCCAG-3' }\end{array}$ & 65 & 121 \\
\hline PVT1 & $\begin{array}{l}\text { PVT1-F } \\
\text { PVT1-R }\end{array}$ & $\begin{array}{l}\text { PVT1-F: 5'-CCTTGGTGTTCCCCTTTTACTGC-3' } \\
\text { PVT1-R: 5'-AACATGGTGAAACCCCGTCTCTAC-3' }\end{array}$ & 65 & 225 \\
\hline NEAT1 & $\begin{array}{l}\text { NEAT1-F } \\
\text { NEAT1-R }\end{array}$ & $\begin{array}{l}\text { NEAT1-F: 5'-CT GGTCTTGTGGAACTGAACTTAGC-3' } \\
\text { NEAT1-R: 5'-AACTCCACATCACTCCTCAGACCAC-3' }\end{array}$ & 65 & 184 \\
\hline PCA3 & $\begin{array}{l}\text { PCA3-F } \\
\text { PCA3-R }\end{array}$ & $\begin{array}{l}\text { PCA3-F: 5'-CCTT CGT GTTGCTGCCTAAT AT G-3' } \\
\text { PCA3-R: 5'-TTTGAGGTCTTGAGAATGGGCAC-3' }\end{array}$ & 65 & 228 \\
\hline Integrin & $\begin{array}{l}\text { Integrin-F } \\
\text { Integrin- } \mathrm{R}\end{array}$ & $\begin{array}{l}\text { Integrin-F: 5'-TGTTTACCACTGATGCCAAGACTC-3' } \\
\text { Integrin-R: 5'-CAAATTGATGTTTTTCTGGGATAGC-3' }\end{array}$ & 64 & 179 \\
\hline B-catenin & $\begin{array}{l}\text { B-catenin-F } \\
\text { B-catenin- } \mathrm{R}\end{array}$ & $\begin{array}{l}\text { B-catenin-F: 5'-AT CCCACTAATGTCCAGCGTTT G-3' } \\
\text { B-catenin-R: 5'-GTCCTCGTCATTTAGCAGTTTTGTC-3' }\end{array}$ & 65 & 140 \\
\hline GAPDH & $\begin{array}{l}\text { GAPDH-F } \\
\text { GAPDH-R }\end{array}$ & $\begin{array}{l}\text { GAPDH-F: 5'-GCCAAAAGGGTCATCATCTCTCTGC-3' } \\
\text { GAPDH-R: 5'-GGTCACGAGTCCTTCCACGATAC-3' }\end{array}$ & 64 & 183 \\
\hline Bak & $\begin{array}{l}\text { Bak-F } \\
\text { Bak-R }\end{array}$ & $\begin{array}{l}\text { Bak-F: 5'-CGTTTTTTACCGCCATCAGCAG-3' } \\
\text { Bak-R: 5'-ATAGCGTCGGTTGATGTCGTCC-3' }\end{array}$ & 66 & 154 \\
\hline Bax & $\begin{array}{l}\text { Bax-F } \\
\text { Bax-R }\end{array}$ & $\begin{array}{l}\text { Bax-F: 5'-AGGTCTTTTTCCGAGTGGCAGC-3' } \\
\text { Bax-R: 5'-GCGTCCCAAAGTAGGAGAGGAG-3' }\end{array}$ & 65 & 234 \\
\hline
\end{tabular}

\section{Confirmation of Recombinant Plasmid}

The $S$. aureus seh gene presence in pcDNA3.1 (+)-seh recombinant vector was confirmed using PCR and BamHI/EcoRV restriction enzyme double digestion. Consequently, a fragment of $193 \mathrm{bp}$ was observed as PCR product on $1 \%$ agarose gel (data were not displayed), and two $5 \mathrm{~kb}$ and $738 \mathrm{bp}$ fragments were

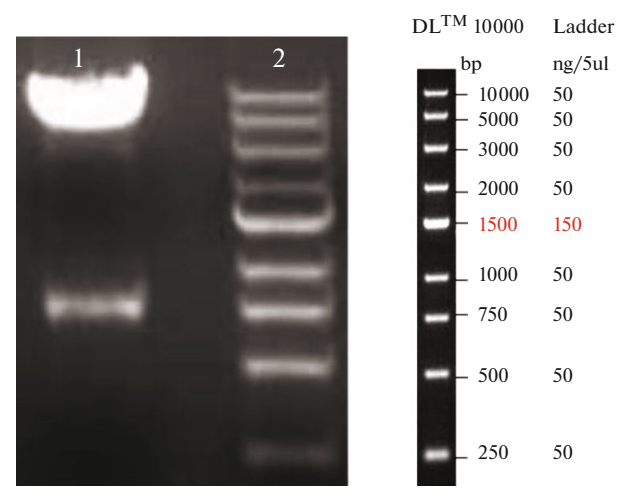

Fig. 1. Confirmation of the recombinant plasmid pcDNA3.1(+)-seh by double restriction endonuclease analysis. Line 1, double restriction endonuclease analysis with $B a m H I / E c o R V$ for pcDNA3.1(+)-seh (recombinant vector); Line 2, DNA size marker. observed after double digestion for pcDNA3.1(+) plasmid and seh gene, respectively (Fig. 1).

\section{Flow Cytometry Assay}

The flow cytometry experiments results indicated the apoptosis and necrosis in ACHN cells that transfected with pcDNA3.1(+)-seh recombinant vector were significantly increased $(p<0.05)$, in comparison with pcDNA3.1(+) plasmid. After seh treatment, the ACHN cells death percentage obviously increased. The flow cytometry data demonstrated that the seh treated ACHN cells were more died (apoptosis) compared to empty plasmid, and this mortality was statistically significant $(p<0.05)$ (Fig. 2a). Similarly, the treatment of PC3 cells results by pcDNA3.1(+)-seh recombinant vector and empty plasmid after flow cytometry analyze, were indicated in Fig. 2b. PC3 cells transfection using recombinant vector indicated more cell death $(p<0.05)$. On the contrary, the apoptosis in HDF cells that were transfected with pcDNA3.1(+)-seh recombinant vector, presented no statistically significant difference in comparison with pcDNA3.1(+) plasmid (Fig. 2c). 

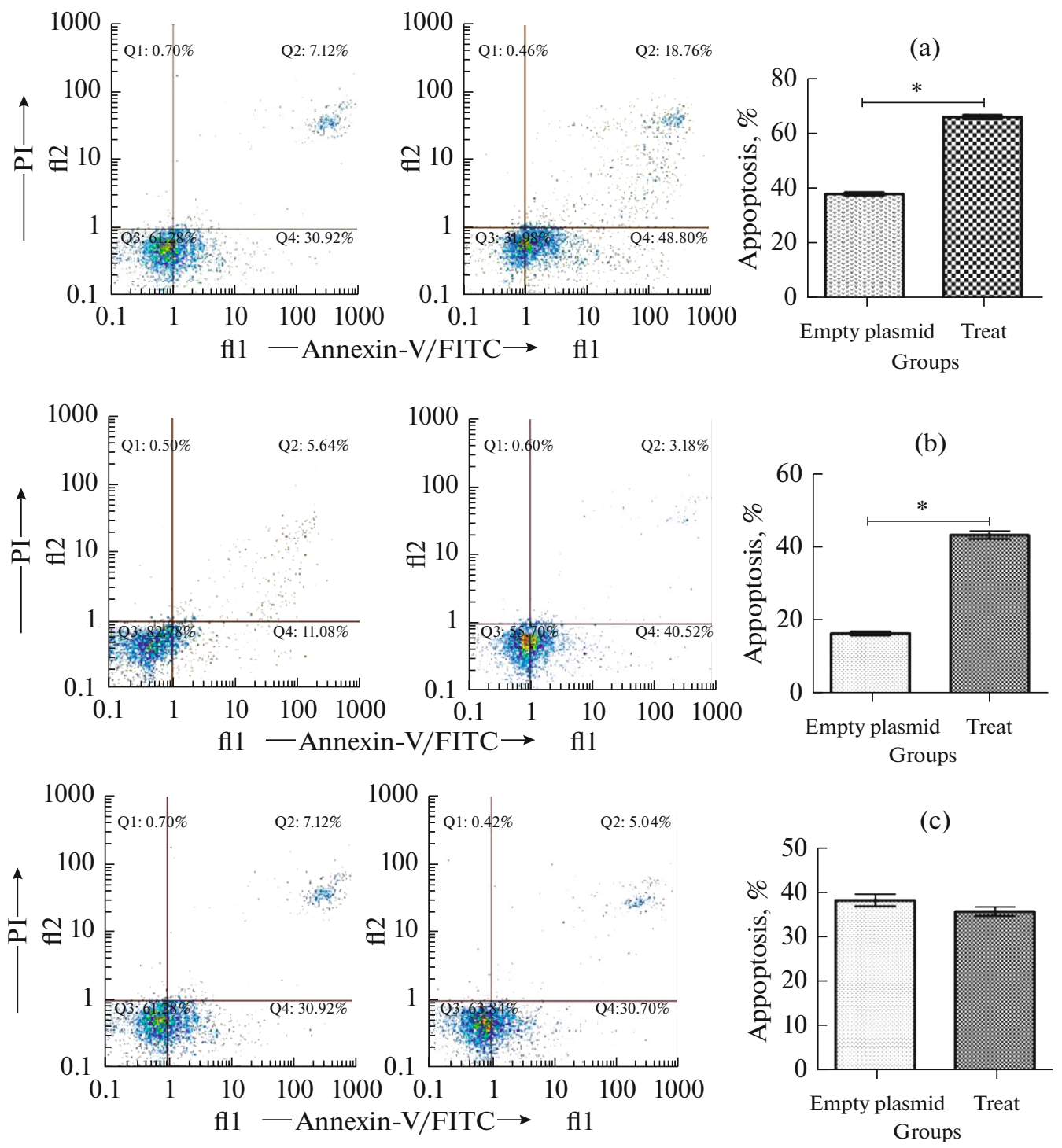

Fig. 2. Detection of cell apoptosis by flow cytometry analysis of surface Annexin V in ACHN (a), PC3 (b) and HDF (c) cells.

\section{Mammalian Expression of seh Gene}

The ACHN, PC3 and HDF cells that were transfected with the recombinant pcDNA3.1 (+)-seh expression vector, were harvested by passing 10 days from transfection. The RT-PCR results indicated that $193 \mathrm{bp}$ fragment were amplified for seh gene, and proposing that the recombinant plasmid was transfected into ACHN, PC3 and HDF cells, successfully (data were not displayed).

\section{qPCR Assay}

We evaluated the PVT1, MALAT1 and GAS5 expression levels in ACHN cell line; PCA3, NEAT1 and GAS5 in PC 3 cells and Bax and Bak genes in HDF cells were transfected with pcDNA3.1(+)-seh in comparison with empty plasmid. Also, the gene expression of $\beta$-catenin and integrin genes were investigated in ACHN and PC3 groups. All information were normalized by the use of GAPDH housekeeping gene. We found a remarkable reduction PVT1 and MALAT1 gene expression using real time RT-PCR in seh treated ACHN cells in comparison with the empty plasmid $(p<0.05$, Figs. 3a, 3b). Also, the statistical analysis demonstrated that the PCA3 and NEAT1 expression in PC3 cells were significantly reduced in pcDNA3.1(+)seh transfected cells in comparison with pcDNA3.1 $(+)$ plasmid ( $p<0.05$, Figs. 3d, 3e). The GAS5 gene expression in ACHN and PC3 cells that were transfected by seh gene of $S$. aureus, were statistically significant increased in comparison with those in empty plasmid (Figs. 3c, 3f). On the contrary, expression levels of $B a x$ and Bak in HDF cell indicated no statistically significant difference between the pcDNA3.1(+)-seh trans- 

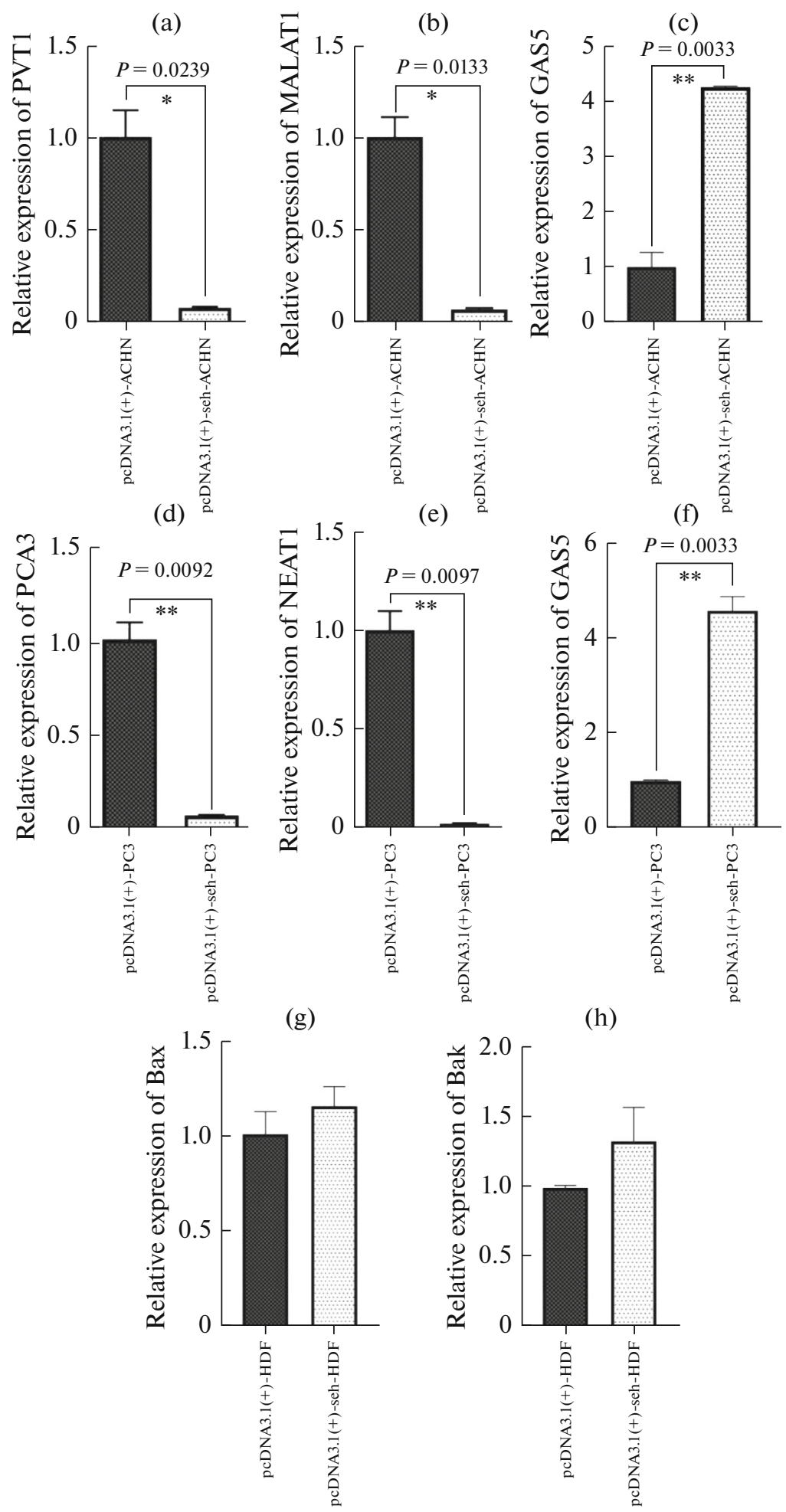

Fig. 3. The expression levels of PVT1, MALAT1 and GAS5 in ACHN cells; PCA3, NEAT1 and GAS5 in PC 3 cells and Bax and Bak in HDF cells were assessed. The data from the real time RT-PCR were normalized versus GAPDH.

fected cells in comparison with the empty plasmid (Figs. 3g, 3h). The comparison of integrin and $\beta$-catenin genes expression indicated statistically significant reduction in seh treated cells (Fig. 4).

\section{DISCUSSION}

Cancer is the second leading cause of death after cardiovascular disease in all over the world, consequently, it is responsible for an estimated 9.6 million 


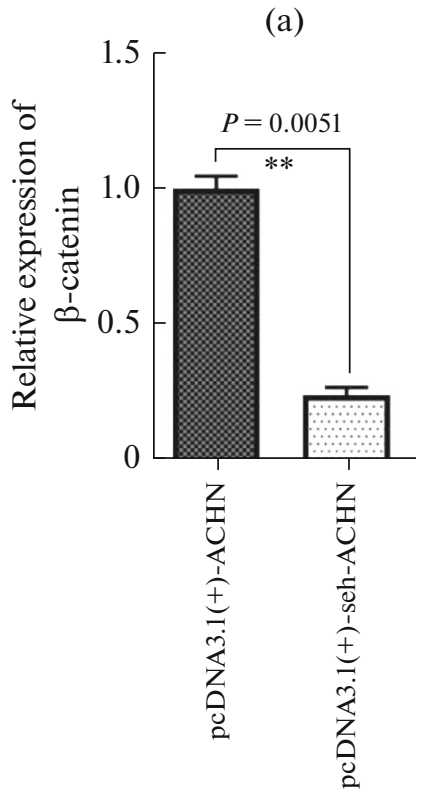

(c)

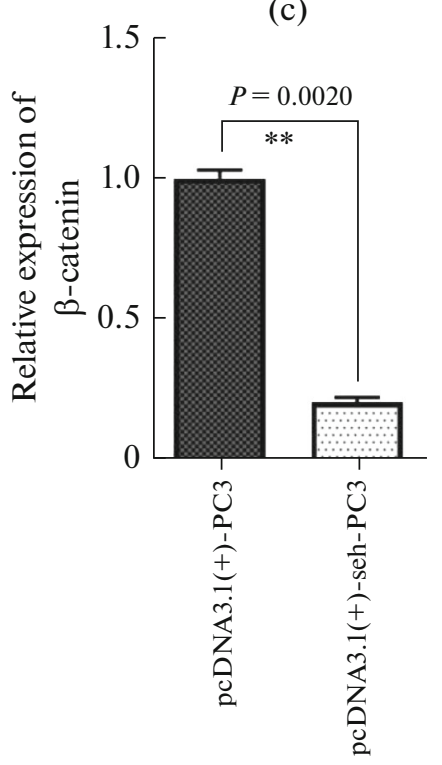

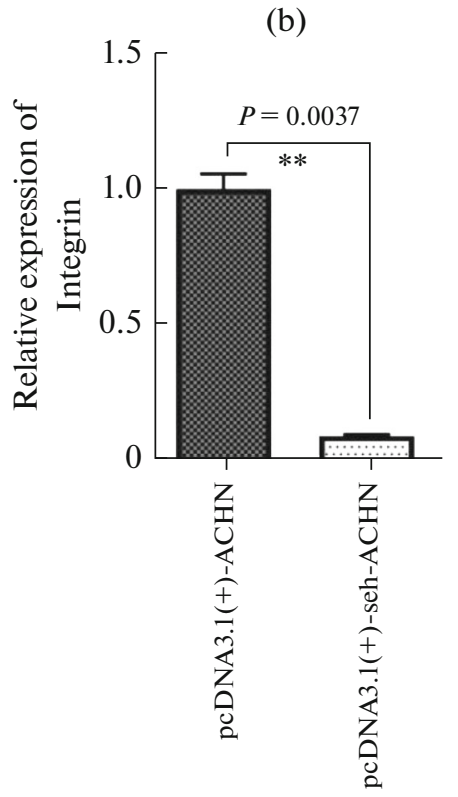

(d)

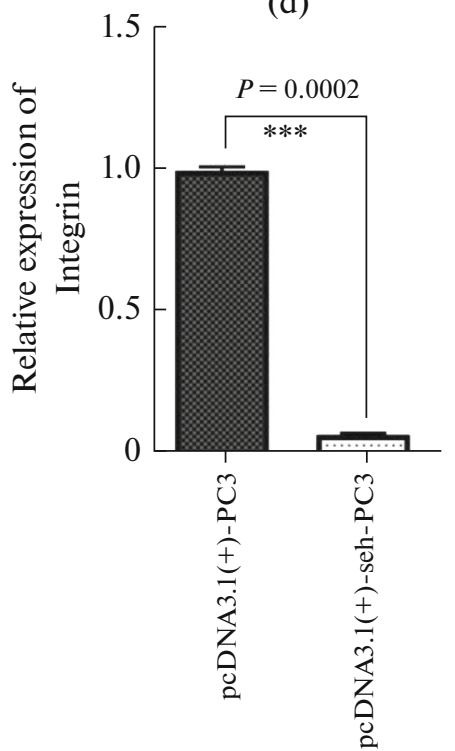

Fig. 4. The expression levels of integrin and $\beta$-catenin genes in pcDNA3.1(+)-seh transfected ACHN (a and b) and PC3 (c and d) cell lines compared with empty plasmid. The data from the real time RT-PCR were normalized versus $G A P D H$.

deaths in 2018. Totally, about 1 from 6 deaths is because of cancer. Approximately one-third of deaths from cancer are caused by the 5 leading behavioral and dietary risk factors as followings: high body mass index, low fruit and vegetable intake, physical inactivity, smoking, and alcohol abuse [15]. Using bacterial toxins in cancer treatment has increased in recent years. Most of these therapies are with respect to the bacterial toxins using in recombinant vectors [16]. Here is a report investigating the SEH toxin of $S$. aureus to Induction of apoptosis and the effects on the expression levels of lncRNAs in PC3 and ACHN cell lines. In conclusion, the 4105 lncRNAs alterations were also investigated that were including mutation, copy number alteration and expressions. After that, the 1 ncRNAs GAS5, PVT1 and MALAT1 were selected from kidney cancer, and GAS5, PCA3 and NEAT1 from prostate cancer. At the molecular level, the effect of recombinant plasmid on the cell lines apoptosis was investigated. The pcDNA3.1(+) mammalian expression vector was applied in order to insert the seh gene encoding. The recombinant plasmid pcDNA3.1(+)seh was constructed and also amplified in a TOP10F chemically competent cell. Two cancer cell lines (ACHN and PC3) and HDF cell, as normal cell, were transfected with pcDNA3.1(+)-seh and pcDNA3.1(+), 
and RT-PCR assay confirmed the seh gene positive expression in seh-transfected cells. Cell death in tested cells was evaluated by the use of the annexin V/PI staining and flow cytometry in order to attain a necrosis and apoptosis measure. More cell death was significantly observed in ACHN and PC3 cells lipofected with pcDNA3.1(+)-seh, compared to control groups $(P<0.05)$. Therefore, this study observations confirmed the significant role of pcDNA3.1(+)-seh in apoptosis. In addition, we evaluated the lncRNAs expression levels after seh treatment. Our findings demonstrated that MALAT1 and PVT1 expression levels in ACHN transfected with pcDNA3.1(+)-seh were significantly reduced in comparison with ACHN transfected with pcDNA3.1(+) vector $(P<0.05)$. The $P C A 3$ and NEAT1 expressions were decreased in sehtreated PC3 cells in comparison with the empty plasmid $(P<0.05)$. It can be therefore discussed that the reduction in these genes expression levels could result in cell death in seh-treated cell lines. Moreover, the $G A S 5$ expression in seh-treated cells increased in both $\mathrm{ACHN}$ and PC3 cell lines.

In one study, the GAS5 overexpression in the A498 RCC cell line, inhibited cell proliferation, induced cell apoptosis and also arrested cell cycle. Furthermore, in another study accomplished by Pickard in 2013, prostate cancer cell lines PC3 and 22Rv1 were transfected with $G A S 5$-encoding pcDNA3 plasmid, and cell survival was investigated, and apoptosis was found to be increased [17, 18]. In this study, we expected that apoptosis would occur after seh-encoding toxin gene expression. As observed in the two above-cited researches, GAS5 overexpression resulted in apoptosis induction, which confirms our hypothesis. In another study (2017), knockdown of NEAT1 by NEAT siRNAs transfection in RCC cell lines suppressed cell proliferation and induced cell apoptosis [19]. In this study, down-regulation of lncRNA NEAT1 was observed after eukaryotic expression of the seh gene in PC 3 cell lines. This confirms the SEH toxin effect on apoptosis induction. Yu (2013) investigated the $S$. aureus toxins SEB and $\alpha$-toxin effects on ECV304 cells. The results indicated apoptosis induction, increasing in TNF- $\alpha$ expression and also caspase 3 and 8 activation in ECV304 cells. These findings propose that SEB and $\alpha$-toxin induce apoptosis throughout the extrinsic apoptosis pathway [20]. In this study, the seh expression effects on PC3 and ACHN cell lines were investigated, and similar results were obtained in terms of apoptosis induction. Also, the integrin and $\beta$-catenin gene expression were reduced, so the cells adhesion were reduced in seh treated cell lines. These results indicated that the $S$. aureus SEH toxin resulted in apoptosis activation throughout pro-apoptotic genes and regulatory lncRNAs in cancer cell lines, but it had no effects on normal HDF cells.

In this study, the tumor progression lncRNAs expression such as MALAT1, PVT1, PCA3 and NEAT1 decreased in the ACHN and $\mathrm{PC} 3$ cancer cell lines, at the time that they were transfected with pcDNA3.1(+)-seh. Furthermore, the GAS5 expression, as a cell apoptosis-inducing lncRNA, was up regulated in the sehtreated ACHN and PC3 cells. pcDNA3.1(+)-seh also increased cell death and apoptosis in transfected cells. In summing up, the $S$. aureus SEH toxin can be applied as a therapeutic bacterial toxin in cancer treatment in the future.

\section{ACKNOWLEDGMENTS}

The authors wish to thanks the staffs of Shahrekord Branch, Islamic Azad University and Cellular and Molecular research center of Shahrekord University of Medical Sciences for their helps.

\section{COMPLIANCE WITH ETHICAL STANDARDS}

The authors declare that they have no conflict of interest. This article does not contain any studies involving animals or human participants performed by any of the authors.

\section{AUTHORS CONTRIBUTIONS}

Abbas Doosti; Contributed to conception and design. Maryam Safarpour-Dehkordi; Contributed to all experimental work, data and statistical analysis, and interpretation of data. Mohammad-Saied Jami; were responsible for overall supervision. All authors read and approved the final manuscript.

\section{ADDITIONAL INFORMATION}

The datasets used and/or analyzed during the current study are available from the corresponding author on reasonable request.

\section{REFERENCES}

1. Drake, C.G., Prostate cancer as a model for tumor immunotherapy, Nat. Rev. Immunol., 2010, vol. 10, no. 8, pp. 580-593.

2. Pernar, C.H., Ebot, E.M., Wilson, K.M., and Mucci, L.A., The epidemiology of prostate cancer, Cold Spring Harbor Perspect. Med., 2018, vol. 12, no. 12, p. a030361.

3. Chen, F.Z. and Zhao, X.K., Prostate cancer: current treatment and prevention strategies, Iran. Red Crescent Med. J., 2016, vol. 15, no. 4, pp. 279-284.

4. Nabi, S., Kessler, E.R., Bernard, B., Flaig, T.W., and Lam, E.T., Cell carcinoma: a review of biology and pathophysiology, F1000Research, 2018, vol. 7, no. 307, pp. 1-10.

5. Medina-Rico, M., Ramos, H.L., Lobo, M., Romo, J., and Prada, J.G., Epidemiology of renal cancer in developing countries: Review of the literature, Can. Urol. Assoc. J., 2018, vol. 12, no. 3, pp. E154-E162.

6. Sánchez-Gastaldo, A., Kempf, E., González, Del., Alba, A., and Duran, I., Systemic treatment of renal cell cancer: A comprehensive review, Cancer Treat. Rev., 2017, vol. 60, pp. 77-89. 
7. Liu, X., Sun, Q., Hou, H., Zhu, K., Wang, Q., Liu, H., et al., The association between BMI and kidney cancer risk: An updated dose-response meta-analysis in accordance with PRISMA guideline, Medicine (Baltimore, $M D, U . S),$.2018 , vol. 97, no. 44, p. e12860.

8. Brodaczewska, K.K., Szczylik, C., Fiedorowicz, M., Porta, C., and Czarnecka, A.M., Choosing the right cell line for renal cell cancer research, Mol. Cancer, 2016, vol. 15, no. 1, p. 83.

9. Forbes, N.S., Engineering the perfect (bacterial) cancer therapy, Nat. Rev. Cancer, 2010, vol. 10, no. 11, pp. 785-794.

10. Pahle, J., Menzel, L., Niesler, N., Kobelt, D., Aumann, J., Rivera, M., et al., Rapid eradication of colon carcinoma by Clostridium perfringens Enterotoxin suicidal gene therapy, BMC Cancer, 2017, vol. 17, no. 1, p. 129.

11. Liu, Y., Chen, W., Ali, T., Alkasir, R., Yin, J., Liu, G., et al., Staphylococcal enterotoxin $\mathrm{H}$ induced apoptosis of bovine mammary epithelial cells in vitro, Toxins (Basel), 2014, vol. 6, no. 12, pp. 3552-3567.

12. Bonetti, A. and Carninci, P., From bench to bedside: The long journey of long noncoding RNAs, Curr. Opin. Syst. Biol., 2017, vol. 3, pp. 119-124.

13. Safarpour Dehkordi, M., Doosti, A., and Arshi, A., Deletion of Salmonella enterica serovar typhimurium sipC gene, Asian Pac. J. Trop. Biomed., 2015, vol. 5, no. 12, pp. 987-991.

14. Seim, I., Jeffery, P.L., Thomas, P.B., Walpole, C.M., Maugham, M., Fung, J.N., et al., Multi-species se- quence comparison reveals conservation of ghrelin gene-derived splice variants encoding a truncated ghrelin peptide, Endocrine, 2016, vol. 52, no. 3 . pp. 609-617.

15. Ott, J.J., Ullrich, A., Mascarenhas, M., and Stevens, G.A., Global cancer incidence and mortality caused by behavior and infection, J. Public Health (Oxford), 2011, vol. 33, no. 2, pp. 223-233.

16. Pahle, J. and Walther, W., Bacterial toxins for oncoleaking suicidal cancer gene therapy, Recent Results Cancer Res., 2016, vol. 209. pp. 95-110.

17. Qiao, H.P., Gao, W.S., Huo, J.X., and Yang, Z.S., Long non-coding RNA GAS5 functions as a tumor suppressor in renal cell carcinoma, Asian Pac. J. Cancer Prev., 2013, vol. 14, no. 2, pp. 1077-1082.

18. Pickard, M.R., Mourtada-Maarabouni, M., and Williams, G.T., Long non-coding RNA GAS5 regulates apoptosis in prostate cancer cell lines, Biochim. Biophys. Acta, 2013, vol. 1832, no. 10, pp. 1613-1623.

19. Ning, L., Li, Z., Wei, D., Chen, H., and Yang, C., LncRNA, NEAT1 is a prognosis biomarker and regulates cancer progression via epithelial-mesenchymal transition in clear cell renal cell carcinoma, Cancer Biomarkers, 2017, vol. 19, no. 1, pp. 75-83.

20. Yu, F.L., Liu, T.T., Zhu, X., Yang, W.X., Zhang, T., Lin, N., et al., Staphylococcal enterotoxin B and $\alpha$ toxin induce the apoptosis of ECV304 cells via similar mechanisms, Mol. Med. Rep., 2013, vol. 8, no. 2, pp. 591-596. 